OPEN ACCESS

Edited by:

Vasilij Goltsev,

Sofia University, Bulgaria

Reviewed by:

Lorenzo Ferroni,

University of Ferrara, Italy

Pengmin Li,

Northwest A\&F University, China

*Correspondence:

Kun Yan

kyan@yic.ac.cn

Shijie Zhao

sjzhao@sdau.edu.cn

Specialty section:

This article was submitted to

Plant Abiotic Stress,

a section of the journal

Frontiers in Plant Science

Received: 14 February 2018

Accepted: 28 March 2018

Published: 07 May 2018

Citation:

Yan K, Han G, Ren C, Zhao S, Wu X and Bian T (2018) Fusarium solani Infection Depressed Photosystem Performance by Inducing Foliage

Wilting in Apple Seedlings.

Front. Plant Sci. 9:479.

doi: $10.3389 /$ fpls.2018.00479

\section{Fusarium solani Infection Depressed Photosystem Performance by Inducing Foliage Wilting in Apple Seedlings}

\author{
Kun Yan ${ }^{1 *}$, Guangxuan Han ${ }^{1}$, Chenggang Ren ${ }^{2}$, Shijie $\mathrm{Zhao}^{3 *}$, Xiaoqing $\mathrm{Wu}^{4}$ and \\ Tiantian Bian ${ }^{1,5}$
}

${ }^{1}$ Key Laboratory of Coastal Environmental Processes and Ecological Remediation, Yantai Institute of Coastal Zone Research, Chinese Academy of Sciences, Yantai, China, ${ }^{2}$ Key Laboratory of Coastal Biology and Bioresource Utilization, Yantai Institute of Coastal Zone Research, Chinese Academy of Sciences, Yantai, China, ${ }^{3}$ State Key Laboratory of Crop Biology, Shandong Agricultural University, Tai'an, China, ${ }^{4}$ Shandong Provincial Key Laboratory of Applied Microbiology, Ecology Institute, Shandong Academy of Sciences, Jinan, China, ${ }^{5}$ School of Life Sciences, Ludong University, Yantai, China

Fusarium fungi are soil-borne pathogens, and the pathological effects on plant photosystems remain unclear. This study aimed to deeply reveal pathological characterization in apple seedlings infected with Fusarium solani by investigating photosystems performance and interaction. Roots were immersed in conidial suspension for inoculation. Thereafter, prompt and delayed chlorophyll a fluorescence and modulated $820 \mathrm{~nm}$ reflection were simultaneously detected. After 30 days of infection, leaf relative water content and dry weight were remarkably decreased by 55.7 and $47.1 \%$, suggesting that the infected seedlings were subjected to Fusarium-induced water deficit stress. PSI reaction center was more susceptible than PSII reaction center in infected seedlings due to greater decrease in the maximal photochemical efficiency of PSI than that of PSII, but PSI reaction center injury was aggravated slowly, as PSII injury could partly protect PSI by restricting electron donation. PSIl donor and acceptor sides were also damaged after 20 days of infection, and the restricted electron donation induced PSII and PSI disconnection by blocking PSI re-reduction. In accordance with greater damage of PSI reaction center, PSI oxidation was also suppressed. Notably, significantly increased efficiency of electron transport from plastoquinone (PQ) to $P S I$ acceptors (REo/ETo) after 20 days of infection suggested greater inhibition on $P Q$ reduction than re-oxidation, and the protection for PSI acceptors might alleviate the reduction of electron transport efficiency beyond $P Q$ upon damaged PSI reaction center. Lowered delayed fluorescence in microsecond domain verified PSIl damage in infected seedlings, and elevated delayed fluorescence in sub-millisecond domain during $P Q$ reduction process conformed to increased REo/ETo. In conclusion, F. solani infection depressed PSII and PSI performance and destroyed their coordination by inducing pathological wilting in apple seedlings. It may be a pathogenic mechanism of Fusarium to induce plant photosystems damage.

Keywords: photosynthetic electron transport, photosystems interaction, plastoquinone, soil-borne pathogen, water deficit 


\section{INTRODUCTION}

Apple replant disease is a serious problem in major applegrowing regions in the world (Mazzola and Manici, 2012). In contrast to abiotic factors such as soil structure and nutrition, the primary origin inducing apple replant disease appears to be soil-borne pathogens, because this disease can be effectively prevented or alleviated by soil disinfection (Yim et al., 2013; Henfrey et al., 2015). To date, some fungi including Fusarium, Rhizoctonia, and Cylindrocarpon have been defined as the main soil-borne pathogens for apple replant disease (Tewoldemedhin et al., 2011a,b,c). Notably, considerable Fusarium fungi exist in replanted apple soil around Bohai Bay in China, and apple seedlings exhibit great susceptibility to these pathogens (Yin et al., 2017). Yantai city is an important apple planting area around Bohai bay in China. At present, about $50 \%$ orchards in Yantai are nearly 30 years old and should be timely reconstructed in these years. Accordingly, young apple trees will be in danger of replant disease in these reconstructed orchards. In fact, replant disease with reduced apple growth and yield have already appeared in some reconstructed orchards in Yantai. Many Fusarium fungi were also found in the soil of these orchards, and the most abundant species was Fusarium solani.

Fusarium fungi can invade plant vascular tissues, impede water transport through xylem by inducing vessel plugging, and lead to foliage wilt. Therefore, wilt symptoms and physiological responses in plants after Fusarium infection are similar to those upon water deficit stress (Wang et al., 2015). Photosynthesis is one of the most important metabolisms for plant growth and its sensitivity to Fusarium infection has been demonstrated in banana, tomato and wheat (Nogues et al., 2002; Dong et al., 2016; Yang et al., 2016). At early stage of Fusarium infection, stomatal limitation was mainly responsible for decreased $\mathrm{CO}_{2}$ assimilation because of stomatal closure under leaf water deficit (Nogues et al., 2002; Yang et al., 2016). The declined $\mathrm{CO}_{2}$ assimilation may induce oxidative stress in chloroplast with elevated generation of reactive oxygen species (ROS) by feedback inhibition of photosynthetic electron transport (Takahashi and Murata, 2008; Sonoike, 2011). As a consequence, carboxylation efficiency and photochemical capacity of PSII were reduced with aggravation of Fusarium infection, suggesting the negative effects on Rubisco and PSII reaction center (Pshibytko et al., 2006; Dong et al., 2016). Up to now, responses of photosystems performance to Fusarium infection have not been deeply explored compared with $\mathrm{CO}_{2}$ assimilation process in photosynthesis. Besides depressed photochemical capacity of PSII reaction center, electron transport capacity at PSII donor and acceptor sides remains to be elucidated in plants infected by Fusarium, and moreover,

\footnotetext{
Abbreviations: $\triangle \mathrm{MR} / \mathrm{MR}_{0}$, the maximal photochemical capacity of PSI; ETo/ABS, quantum yield for electron transport; ETo/TRo, probability that an electron moves further than primary acceptor of PSII; $F_{\mathrm{v}} / F_{\mathrm{m}}$, the maximal quantum yield of

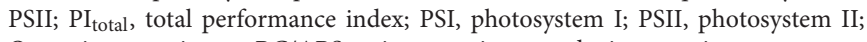
$\mathrm{Q}_{\mathrm{A}}$, primary quinone; $\mathrm{RC} / \mathrm{ABS}$, primary quinone reducing reaction centers per PSII antenna chlorophyll; REo/ABS, quantum yield for reduction of end electron acceptors of PSI; REo/ETo, probability with which an electron from the intersystem electron carriers is transferred to reduce end electron acceptors at the PSI acceptor side; $W_{\mathrm{k}}$, ratio of relative variable fluorescence at $300 \mu \mathrm{s}$ to that at J step.
}

PSI performance and interaction between PSII and PSI are also largely unknown. To date, although the inhibited growth and photosynthesis have been reported in apple grown in replant soil, the direct effects of Fusarium infection on apple photosystems are still unclear (Wang et al., 2014; Henfrey et al., 2015).

Recently, a simultaneous measurement of prompt chlorophyll $a$ fluorescence (PF), delayed chlorophyll $a$ fluorescence (DF), and modulated $820 \mathrm{~nm}$ reflection (MR) has been developed, and this technique allows collection and correlation of complementary information for diagnosing PSII and PSI performance and investigating characterization of photosynthetic electron transport (Strasser et al., 2010; Goltsev et al., 2012; Oukarroum et al., 2013, 2016a; Gao et al., 2014). In this study, we aimed to explore whether F. solani could induce water deficit stress in apple seedlings, and then investigate the responses of PSII and PSI performance and their interaction through simultaneously detecting PF, DF, and MR. According to the studies on drought stress, PSII reaction center is commonly more sensitive than PSI reaction center in crops except the contrary result in some trees with tremendous leaf water deficit after long severe drought stress, and the limitation of electron transport from PSII can retard ROS production by preventing over-reduction of PSI acceptor side (Goltsev et al., 2012; Huang et al., 2013; Campos et al., 2014; Zivcak et al., 2014b; Zhang S.B. et al., 2016). Therefore, we hypothesized that infection of $F$. solani could depress PSII and PSI performance and destroy their coordination by inducing foliage wilting in apple seedlings, and greater vulnerability of PSII could help alleviate the detrimental effects on PSI. This study can deepen the knowledge of pathological characterization in apple with Fusarium infection and assist in revealing the source of apple replant disease in Yantai.

\section{MATERIALS AND METHODS}

\section{Plant Material and Inoculation}

Apple (Malus hupehensis Rehd.) seeds were stored in sand at $4^{\circ} \mathrm{C}$ for 30 days to sprout, and the sprouts were sown in nursery plates filled with sand. The sprouts were watered with Hoagland solution $(\mathrm{pH}$ 5.7) and placed in artificial climatic chambers (Huier, China). In this study, the photon flux density was approximately $200 \mu \mathrm{mol} \mathrm{m}{ }^{-2} \mathrm{~s}^{-1}(12 \mathrm{~h}$ per day from 07:00 to 19:00) in the chambers, and day/night temperature and humidity were controlled at $25 / 18^{\circ} \mathrm{C}$ and $65 \%$. After 2 months, uniform seedlings which developed six leaves were selected for inoculation.

Rhizosphere soil was sampled in replant apple orchards in Yantai $\left(37^{\circ} 11^{\prime} \mathrm{N}, 121^{\circ} 11^{\prime} \mathrm{E}\right)$, and these replant orchards were reconstructed from 30-year-old orchards. In these replant orchards, apple trees were 5-year-old in 2016 and exhibited obvious replant disease with reduced growth and yield. Fungi were isolated from the rhizosphere soil and purified by plate culture with Martin medium. Genome DNA of the fungi was extracted through CTAB method, and PCRs were carried out 
with ITS1 and ITS4 as the primers. PCR products were used for sequencing, and fungi were identified by blasting the sequences in NCBI. The results suggested that many fungi belonged to $F$. solani, as their sequence identity with reported $F$. solani (accession: MF467479.1) reached 100\%. A strain of F. solani exhibiting rapid growth in potato dextrose agar medium was designated as F. solani A07, and used for apple infection experiment in our study. It has been preserved as a patented strain in China General Microbiological Culture Collection Center (CGMCC), and the preservation number is CGMCC No. 13187.

Fusarium solani A07 was cultured on a potato dextrose agar medium at $28^{\circ} \mathrm{C}$ in the dark for 7 days, and discs of fungus-containing agar was excised from the culture margins and inoculated into Erlenmeyer flasks containing potato dextrose agar medium. The flasks were incubated for 7 days at $28^{\circ} \mathrm{C}$ with rotary shaking at $180 \mathrm{rpm}$, and then mycelial fragments were removed by filtering through sterile cheesecloth to obtain conidial suspension. The conidial suspension was diluted to $4 \times 10^{6}$ spore $\mathrm{ml}^{-1}$ using sterile water, and then the roots of apple seedlings were immersed in the conidial suspension for $2 \mathrm{~h}$ for inoculation, while the roots of control seedlings were immersed in sterile water for $2 \mathrm{~h}$. After inoculation, the seedlings were planted in plastic pots filled with sterilized soil and placed in artificial climatic chambers under the same culture conditions as those before inoculation. Five replicate seedlings were respectively selected from inoculated and control groups for each experiment, and the newly expanded leaves were used for the measurements.

\section{Measurements of Leaf Biomass, Proline, and Relative Water Contents}

Fresh leaves were harvested and weighed (fresh weight, FW). Subsequently, they were immersed in distilled water for $4 \mathrm{~h}$ at room temperature to determine saturated fresh weight (SW). At last, the leaves were dried completely in an oven at $70^{\circ} \mathrm{C}$ and weighed (dry weight, DW). Relative water content (RWC) was calculated as: $\mathrm{RWC}=(\mathrm{FW}-\mathrm{DW}) /(\mathrm{SW}-\mathrm{DW}) \times 100 \%$.

Proline content was measured by using ninhydrin coloration method (Yan et al., 2012b). Dry plant powder $(0.1 \mathrm{~g})$ was homogenized with $5 \mathrm{ml}$ of sulfosalicylic acid (3\% w/v). After centrifugation, the supernatant $(2 \mathrm{ml})$ was incubated with glacial acetic acid $(2 \mathrm{ml})$ and ninhydrin reagent $(3 \mathrm{ml})$ at $100^{\circ} \mathrm{C}$ for $40 \mathrm{~min}$. After cooling, $5 \mathrm{ml}$ of toluene was added to the mixture, and then the absorbance of chromophore-containing toluene was recorded at $520 \mathrm{~nm}$. A standard curve was plotted by using known concentrations of proline to determine leaf proline content. The absorbance was measured by using a UV-1800 spectrophotometer (Shimadzu, Japan).

\section{Simultaneous Measurements of PF, DF, and MR Transients}

The measurements were made by using a multifunctional plant efficiency analyzer (MPEA, Hansatech, United Kingdom), and the operating mechanism of this instrument has been elucidated in detail (Strasser et al., 2010). This powerful instrument has been widely used for investigating PSII and PSI performance and photosynthetic electron transport process in recent years (Strasser et al., 2010; Goltsev et al., 2012; Oukarroum et al., 2013, 2016a; Gao et al., 2014; Yan et al., 2015). The leaves were adapted in dark for $30 \mathrm{~min}$ before measurement. Thereafter, the leaves were illuminated with $1 \mathrm{~s}$ red light $\left(627 \mathrm{~nm}, 5000 \mu \mathrm{mol}\right.$ photons $\mathrm{m}^{-2} \mathrm{~s}^{-1}$ ) and subsequently with $10 \mathrm{~s}$ far red light $\left(735 \mathrm{~nm}, 200 \mu \mathrm{mol}\right.$ photons $\left.\mathrm{m}^{-2} \mathrm{~s}^{-1}\right)$. $\mathrm{PF}, \mathrm{DF}$, and MR transients were simultaneously recorded in the first $1 \mathrm{~s}$ illumination with red light, and MR data were also detected in the following $10 \mathrm{~s}$ illumination with far red.

Oxidation of PSI reaction center is known to cause an increase in absorbance in $800-850 \mathrm{~nm}$ range, and monitoring $820 \mathrm{~nm}$ reflection is a very convenient way to follow the redox state of PSI reaction center under continuous light (Schansker et al., 2003). A modulated light source built in the measuring head of MPEA allowed the measurement of the kinetics of light-induced absorption changes at $820 \mathrm{~nm}$ (Strasser et al., 2010). $\mathrm{MR}_{0}$ is the value at onset of red light illumination, when PSI reaction center is in reduced form (0.7 ms, the first reliable MR measurement; Strasser et al., 2010). After far red illumination for $10 \mathrm{~s}$, PSI reaction center was completely oxidized, and the relative difference of $820 \mathrm{~nm}$ reflection between the maximal oxidized and reduced PSI reaction center $\left(\triangle \mathrm{MR} / \mathrm{MR}_{0}\right)$ was used as an index of the maximal photochemical capacity of PSI (Schansker et al., 2003; Yan et al., 2012a).

Prompt chlorophyll $a$ fluorescence transients were quantified according to JIP test by using the following original data: (1) fluorescence intensity at $20 \mathrm{~s}\left(F_{\mathrm{o}}\right.$, when all reaction centers of PSII are open); (2) the maximal fluorescence intensity $\left(F_{\mathrm{m}}\right.$, when all reaction centers of PSII are closed); and (3) fluorescence intensities at $300 \mu \mathrm{s}$ (K step), $2 \mathrm{~ms}$ (J step), and $30 \mathrm{~ms}$ (I step). Using these original data, some parameters can be calculated for quantifying photosynthetic electron transport (Strasser et al., 2010). The parameters and formulae are listed in Table 1.

All redox reactions of the photosynthetic electron transport are reversible. The accumulation of electrons in the electron transport chain between PSII and PSI leads to back electron transfer and charge recombination in PSII reaction center, resulting in re-excitation of reaction center and repopulation of excited chlorophyll state of PSII antenna. The light emission from repopulated excited chlorophyll is delayed fluorescence (Goltsev et al., 2009). In order to exclude PF interference under the light, simultaneous measurement of DF requires alteration of light and dark intervals, and DF decay signals are continuously recorded in dark intervals (Goltsev et al., 2009; Strasser et al., 2010). In this study, DF signals in microsecond and sub-millisecond domains were collected, respectively, at 20 and $200 \mu \mathrm{s}$ after turning off actinic light for constructing DF transients.

\section{Statistical Analysis}

One-way ANOVAs were carried out by using SPSS 16.0 (SPSS Inc., Chicago, IL, United States) for all sets of data. The values 
TABLE 1 | Formulae and terms used in the analysis of OJIP fluorescence transient.

\begin{tabular}{|c|c|}
\hline \multicolumn{2}{|c|}{ Data extracted from the recorded fluorescence transient OJIP } \\
\hline$F_{\mathrm{t}}$ & $\begin{array}{l}\text { Fluorescence intensity at time } t \text { after onset } \\
\text { of actinic illumination }\end{array}$ \\
\hline$F_{0}=F_{20 \mu \mathrm{s}}$ & Minimal recorded fluorescence intensity \\
\hline$F_{\mathrm{k}}=F_{300 \mu \mathrm{s}}$ & Fluorescence intensity at $300 \mu \mathrm{s}$ \\
\hline$F_{\mathrm{J}}=F_{2} \mathrm{~ms}$ & Fluorescence intensity at the $\mathrm{J}$ step \\
\hline$F_{1}=F_{30 \mathrm{~ms}}$ & Fluorescence intensity at the I step \\
\hline$F_{\mathrm{m}}$ & Maximal fluorescence intensity \\
\hline \multicolumn{2}{|c|}{ Fluorescence parameters derived from the extracted data } \\
\hline$V_{\mathrm{t}}=\left(F_{\mathrm{t}}-F_{\mathrm{o}}\right) /\left(F_{\mathrm{m}}-F_{\mathrm{o}}\right)$ & Relative variable fluorescence at time $t$ \\
\hline $\begin{array}{l}V_{k}=\left(F_{k}-\text { ctron transport process }\right. \\
\left.\text { in recent years } F_{0}\right) /\left(F_{m}-F_{0}\right)\end{array}$ & $\begin{array}{l}\text { Relative variable fluorescence intensity at } \mathrm{K} \\
\text { step }\end{array}$ \\
\hline$V_{J}=\left(F_{J}-F_{0}\right) /\left(F_{m}-F_{0}\right)$ & $\begin{array}{l}\text { Relative variable fluorescence intensity at } J \\
\text { step }\end{array}$ \\
\hline$V_{1}=\left(F_{1}-F_{0}\right) /\left(F_{m}-F_{0}\right)$ & $\begin{array}{l}\text { Relative variable fluorescence intensity at I } \\
\text { step }\end{array}$ \\
\hline$W_{\mathrm{k}}=\left(F_{\mathrm{k}}-F_{\mathrm{o}}\right) /\left(F_{\mathrm{J}}-F_{\mathrm{o}}\right)$ & $\begin{array}{l}\text { Ratio of relative variable fluorescence at } \mathrm{K} \\
\text { step to that at } \mathrm{J} \text { step }\end{array}$ \\
\hline \multicolumn{2}{|c|}{ Biological parameters derived from the fluorescence parameters } \\
\hline$F_{\mathrm{v}} / F_{\mathrm{m}}=1-F_{\mathrm{o}} / F_{\mathrm{m}}$ & $\begin{array}{l}\text { Maximum quantum yield for primary } \\
\text { photochemistry }\end{array}$ \\
\hline $\mathrm{ETO} / \mathrm{TRo}=1-V_{J}$ & $\begin{array}{l}\text { Probability that an electron moves further } \\
\text { than } Q_{A}\end{array}$ \\
\hline $\mathrm{REO} / \mathrm{ETO}=\left(1-V_{\mathrm{I}}\right) /\left(1-V_{\mathrm{J}}\right)$ & $\begin{array}{l}\text { Probability with which an electron from the } \\
\text { intersystem electron carriers is transferred } \\
\text { to reduce end electron acceptors at the PSI } \\
\text { acceptor side }\end{array}$ \\
\hline $\mathrm{ETo} / \mathrm{ABS}=\left(F_{\mathrm{m}}-F_{\mathrm{J}}\right) / F_{\mathrm{m}}$ & Quantum yield for electron transport \\
\hline $\mathrm{REo} / \mathrm{ABS}=\left(F_{\mathrm{m}}-F_{\mathrm{l}}\right) / F_{\mathrm{m}}$ & $\begin{array}{l}\text { Quantum yield for reduction of end electron } \\
\text { acceptors of PSI }\end{array}$ \\
\hline $\mathrm{RC} / \mathrm{ABS}=\left(V_{\mathrm{J}} / 4 V_{\mathrm{k}}\right) F_{\mathrm{v}} / F_{\mathrm{m}}$ & $\begin{array}{l}Q_{A} \text { reducing reaction centers per } P S I I \\
\text { antenna chlorophyll }\end{array}$ \\
\hline $\begin{array}{l}\left.\mathrm{P}\right|_{\text {total }}=\mathrm{RC} / \mathrm{ABS} \cdot[\mathrm{TRO} /(\mathrm{ABS}-\mathrm{TRO})] \cdot \\
{[\mathrm{ETO} /(\mathrm{TRo}-\mathrm{ETO})] \cdot[\mathrm{REO} /(\mathrm{ETO}-\mathrm{REO})]}\end{array}$ & Total performance index \\
\hline
\end{tabular}

$Q_{A}$, primary quinone; PSI, photosystem I; PSII, photosystem II.

presented are the mean of samples collected from five replicate seedlings in inoculated and control groups. The comparisons of means were determined through least significant difference test, and the differences were considered significant at $P<0.05$.

\section{RESULTS}

\section{Leaf Biomass, Relative Water Content, and Proline Content}

After 30 days of infection, FW, DW, and RWC in the leaves were tremendously decreased by $55.3,47.1$, and $55.7 \%$, and leaf proline content was remarkably elevated by 7.8 folds (Table 2 ).

\section{Total Performance Index and the Maximal Photochemical Efficiency of PSII and PSI}

The definition of $\mathrm{PI}_{\text {total }}$ and $F_{\mathrm{v}} / F_{\mathrm{m}}$ is listed in Table 1. During the first 5 days of infection, $\mathrm{PI}_{\text {total }}, F_{\mathrm{v}} / F_{\mathrm{m}}$ and $\Delta \mathrm{MR} / \mathrm{MR}_{0}$ were not affected (Figures 1A-C). After 10 days of infection, $\mathrm{PI}_{\text {total }}, \Delta \mathrm{MR} / \mathrm{MR}_{0}$ and the ratio of $\Delta \mathrm{MR} / \mathrm{MR}_{0}$ to $F_{\mathrm{v}} / F_{\mathrm{m}}$ were significantly decreased in line with slightly lowered $F_{\mathrm{V}} / F_{\mathrm{m}}$, however, the decrease in $F_{\mathrm{v}} / F_{\mathrm{m}}$ appeared to be faster than $\triangle \mathrm{MR} / \mathrm{MR}_{0}$ in the following days (Figure 1). From infection for 10 days to 30 days, decrease in the ratio of $\Delta \mathrm{MR} / \mathrm{MR}_{0}$ to $F_{\mathrm{v}} / F_{\mathrm{m}}$ was curtailed from 24.5 to $20.6 \%$ (Figure 1D), which verified the faster decrease in $F_{\mathrm{V}} / F_{\mathrm{m}}$ than $\Delta \mathrm{MR} / \mathrm{MR}_{0}$.

\section{Transients of PF, DF, and MR}

No obvious change occurred in PF transient except for a slight elevation of $\mathrm{J}$ step after 10 days of infection (Figure 2A). When the infection was prolonged to 20 and 30 days, J step was significantly elevated, and a clear $\mathrm{K}$ step appeared in the transients (Figure 2A).

As shown in Figure 2B, MR signals are presented by $M R / M_{0}$ ratio, where $M R_{0}$ is the value at onset of actinic illumination (at $0.7 \mathrm{~ms}$ ). Decrease in $\mathrm{MR} / \mathrm{MR}_{0}$ from $\mathrm{MR}_{0}$ to the minimal value $\left(\mathrm{MR}_{\min }\right)$ reflects PSI oxidation process. $\mathrm{MR}_{\min }$ is a transitory steady state with equal oxidation and re-reduction rate of PSI reaction center. Subsequently, increase of $M R / M R_{0}$ from $M R_{\min }$ to the maximal level $\left(\mathrm{MR}_{\max }\right)$ indicates PSI re-reduction. After 10 days of infection, MR transient was obviously affected, as PSI oxidation process was shortened. Besides shortened PSI oxidation process, PSI re-reduction was also slowed when the infection was prolonged to 20 days (Figure 2B).

Delayed chlorophyll a fluorescence signals include microsecond and sub-millisecond components, and they are presented by $\mathrm{DF}_{t} / \mathrm{DF}_{0.3} \mathrm{~ms}$ and $\mathrm{DF}_{\mathrm{t}} / \mathrm{DF}_{3} \mathrm{~ms}$, respectively. $\mathrm{I}_{1}(7 \mathrm{~ms})$ and $\mathrm{I}_{2}(50 \mathrm{~ms})$ are two peaks in DF transients (Figures 2C,D). $I_{1}$ peak appears due to the accumulation of $\mathrm{S}_{3} \mathrm{Z}^{+} \mathrm{P}_{680 \mathrm{Q}^{-}}{ }^{-}$state, because $\mathrm{S}_{3}$ state exhibits the highest DF signal during the S-state cycle of oxygen evolving complex (OEC), and $\mathrm{I}_{2}$ is related to the prolonged reopening of PSII reaction centers by electron transfer from reduced $\mathrm{Q}_{\mathrm{B}}$ to plastoquinone (PQ; Goltsev et al., 2009). $\mathrm{DF}_{0.3 \mathrm{~ms}}$ is the initial microsecond delayed fluorescence signal at $0.3 \mathrm{~ms}$, and $\mathrm{DF}_{3} \mathrm{~ms}$ is the initial sub-millisecond delayed fluorescence signal at $3 \mathrm{~ms}$. The microsecond and sub-millisecond DF transients were not obviously affected after 10 days of infection except for a slight decrease of $\mathrm{I}_{1}$ peak in microsecond DF transient (Figure 2C). After 20 and 30 days of infection, $I_{1}$ and $I_{2}$ peaks in microsecond DF transients were significantly lowered, whereas significant

TABLE 2 | Fresh weight (FW), dry weight (DW), relative water content, and proline content in the leaves of apple seedlings after 30 days of infection with Fusarium solani.

\begin{tabular}{|c|c|c|c|c|}
\hline Treatments & FW per leaf $(g)$ & DW per leaf $(g)$ & Relative water content (\%) & Proline content ( $\left.\mathrm{mg} \mathrm{g}^{-1} \mathrm{DW}\right)$ \\
\hline Control plants & $0.085 \pm 0.005 a$ & $0.034 \pm 0.007 a$ & $88 \pm 1.5 a$ & $0.38 \pm 0.05 a$ \\
\hline Infected plants & $0.038 \pm 0.009 b$ & $0.018 \pm 0.005 b$ & $39 \pm 2.1 b$ & $3.35 \pm 0.08 b$ \\
\hline
\end{tabular}

Data in the table indicate the mean of five replicates $( \pm S D)$. Within each column, mean values followed by the different letters are significantly different at $P<0.05$. 


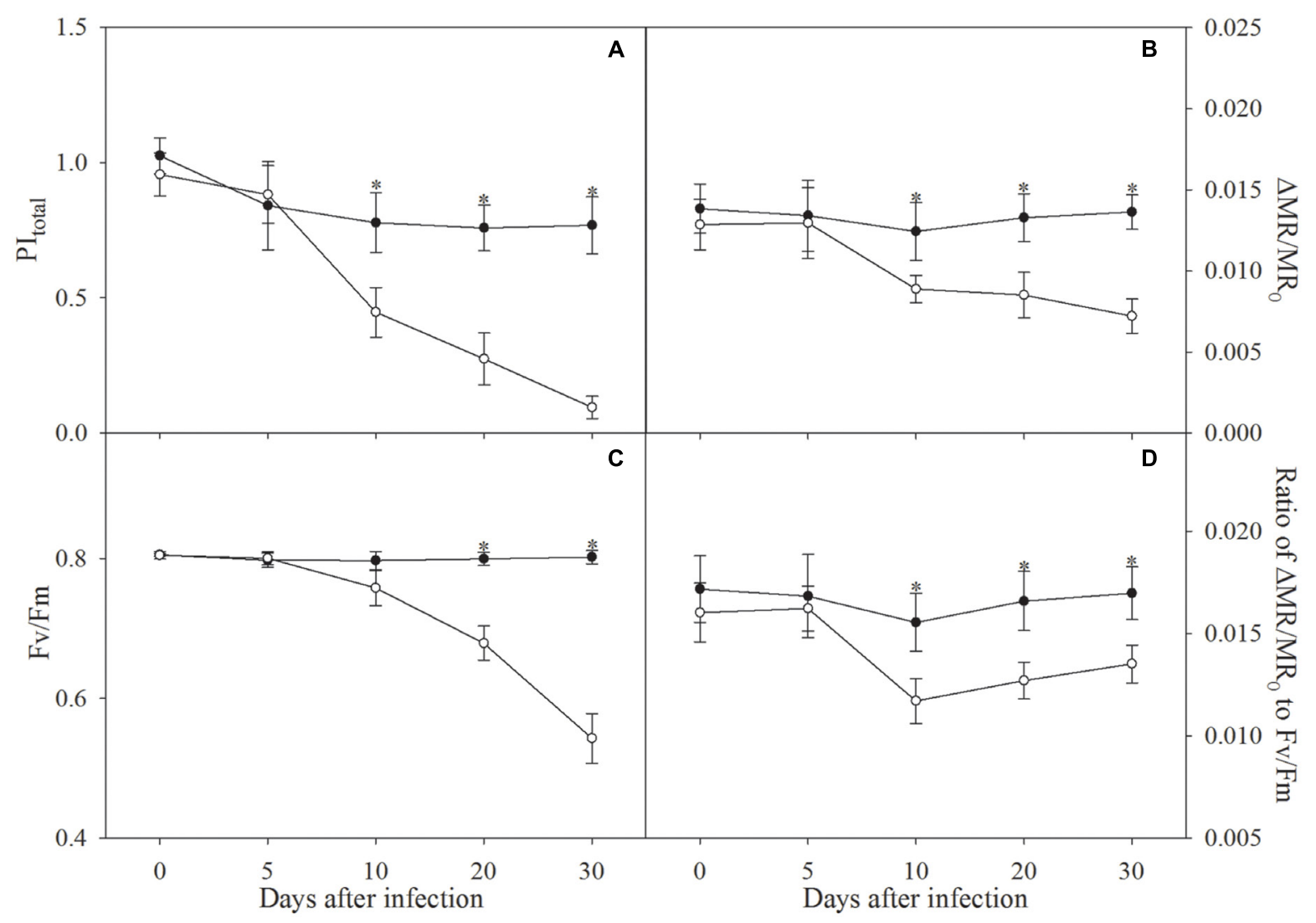

FIGURE 1 | Total performance index ( $\left.\mathrm{Pl}_{\text {total }}, \mathbf{A}\right)$, the maximal photochemical efficiency of PSI $\left(\Delta M R / M R_{0}, \mathbf{B}\right)$, the maximal photochemical efficiency of PSII $\left(F_{\mathrm{v}} / F_{\mathrm{m}}, \mathbf{C}\right)$, and ratio of $\Delta \mathrm{MR} / \mathrm{MR}_{0}$ to $F_{\mathrm{v}} / F_{\mathrm{m}}(\mathbf{D})$ in control plants (closed symbols) and plants infected by Fusarium solani (open symbols). Data in the figure indicate the mean of five replicates $( \pm S D)$, and asterisk indicates significant difference at $P<0.05$.

elevation of $\mathrm{I}_{2}$ peak was noted in sub-millisecond DF transients (Figures 2C,D).

\section{Characterization of Photosynthetic Electron Transport According to JIP Test}

The definition of $W_{\mathrm{k}}$, RC/ABS, ETo/TRo, ETo/ABS, REo/ETo, and $\mathrm{REo} / \mathrm{ABS}$ is listed in Table 1. Significant increase in $W_{\mathrm{k}}$ and REo/ETo did not occur in apple seedlings until the infection was prolonged to 20 days (Figures 3A,E). Decrease in RC/ABS, ETo/TRo, ETo/ABS, and REo/ABS was noted after 10 days of infection, and the decrease became significant after 20 days of infection (Figures 3B-D,F).

\section{PSI Oxidation and Re-reduction Amplitude and $\mathrm{I}_{2} / \mathrm{I}_{1}$}

Significant decrease in $M R_{0}-M_{\min }$ and $M R_{\max }-\mathrm{MR}_{\min }$ was observed, respectively, after 10 and 20 days of infection (Figures 4A,B). Since infection for 10 days, the ratio of $\mathrm{MR}_{0^{-}}$ $\mathrm{MR}_{\mathrm{min}}$ in infected seedlings to control seedlings did not change, whereas the ratio of $M R_{\max }-M_{\min }$ in infected seedlings to control seedlings was significantly lowered (Figures 4E,F). $\mathrm{I}_{2} / \mathrm{I}_{1}$ in both microsecond and sub-millisecond domains was significantly increased after 20 days of infection (Figures 4C,D).
The ratio of $I_{2} / I_{1}$ in infected seedlings to control seedlings was significantly higher after infection for 20 and 30 days than the value after 10 days of infection (Figures $4 \mathbf{G}, \mathbf{H}$ ).

\section{DISCUSSION}

Fusarium fungi can impede water transport through xylem by inducing vessel plugging and then bring about pathological foliage wilting in plants (Wang et al., 2015). Accordingly, large decrease in leaf RWC suggested that F. solani induced pathological wilting in apple seedlings, and thus, the infected seedlings were subjected to severe water deficit stress. This result was further verified by significant increase in leaf proline content (Table 2), because proline is a crucial osmolyte in plant tissues for resisting water deficit (Ashraf and Foolad, 2007; Yan et al., $2012 b)$. Thus, as with other plants, leaf biomass was reduced in apple seedlings by F. solani (Table 2; Wang et al., 2015; Dong et al., 2016; Yang et al., 2016).

Performance index can sensitively reflect photosynthetic apparatus function (Zivcak et al., 2008, 2014c), and significant decrease in $\mathrm{PI}_{\text {total }}$ after 10 days of infection indicated that photosynthetic apparatus was injured due to pathological foliage wilting (Figure 1A). PSI reaction center exhibited higher 

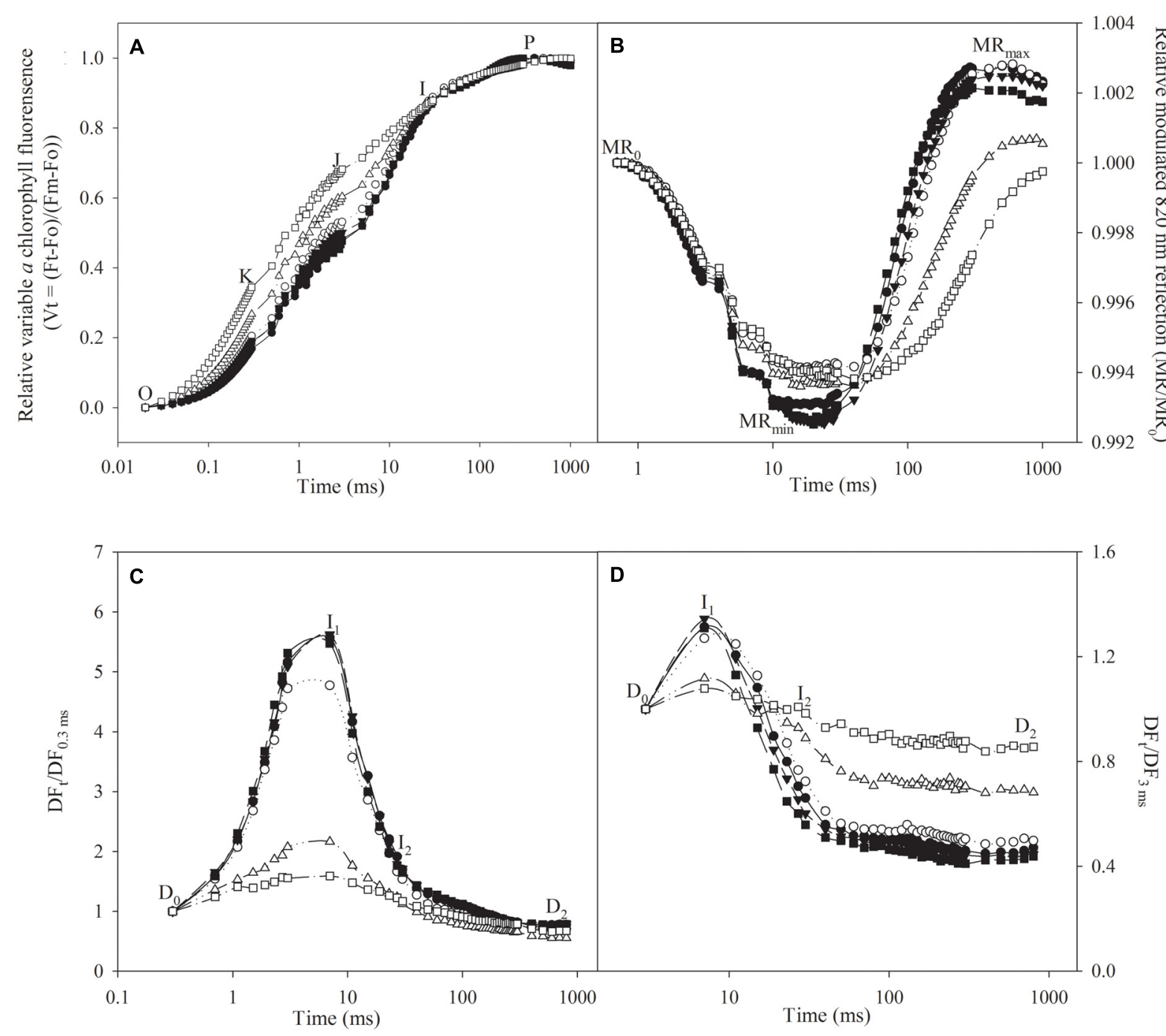

FIGURE 2 | Transients of prompt chlorophyll a fluorescence (A), modulated $820 \mathrm{~nm}$ reflection (B), and delayed chlorophyll a fluorescence in microsecond domain (C), and sub-millisecond domain (D) in control plants (closed symbols) and plants infected by Fusarium solani (open symbols) for 10 days (circles), 20 days (triangles), and 30 days (squares). $\mathrm{O}, \mathrm{K}, \mathrm{J}, \mathrm{I}$, and $\mathrm{P}$ indicate the specific steps in chlorophyll a fluorescence transient. $\mathrm{MR}_{0}$ is the value of modulated 820 nm reflection at the onset of red light illumination ( $0.7 \mathrm{~ms}$, the first reliable MR measurement). $\mathrm{MR}_{\min }$ and $\mathrm{MR}_{\max }$ indicate the maximal point during PSI oxidation and the maximal point during PSI re-reduction, respectively. $D_{0}, I_{1}, I_{2}$, and $D_{2}$ indicate initial point, the first $(7 \mathrm{~ms})$ and second (50 ms) maximal peaks and minimum point in delayed chlorophyll a fluorescence curves. $\mathrm{DF}_{0.3 \mathrm{~ms}}$ is the initial microsecond delayed fluorescence signal at $0.3 \mathrm{~ms}$, and DF 3 ms is the initial sub-millisecond delayed fluorescence signal at $3 \mathrm{~ms}$. The signals were plotted on a logarithmic time scale. Each curve is the average of five replicates.

susceptibility than PSII reaction center due to significant decrease of $\triangle \mathrm{MR} / \mathrm{MR}_{0}$ with slight change in $F_{\mathrm{V}} / F_{\mathrm{m}}$ after 10 days of infection (Figures 1B,C), and the greater damage on PSI reaction center was also corroborated by significant decrease in ratio of $\Delta \mathrm{MR} / \mathrm{MR}_{0}$ to $F_{\mathrm{V}} / F_{\mathrm{m}}$ since infection for 10 days (Figure 1D). However, PSI commonly has stronger drought tolerance than PSII in crops (Goltsev et al., 2012; Zivcak et al., 2014b; Zhang S.B. et al., 2016). In our opinion, this common finding derives from crops upon moderate or mild drought stress, and may not appear in apple under severe water deficit stress with tremendous leaf water loss. Huang et al. (2013) also reported that severe drought stress led to greater PSI damage compared with PSII in trees with large leaf water loss, and proposed that PSI sensitivity resulted from its slower recovery mainly due to inhibited cyclic electron flow around PSI. As a traditional viewpoint, PSII photoinhibition can protect PSI by restricting electron flow to PSI acceptor side for reducing ROS production (Sonoike, 2011; Zivcak et al., 2014a; Oukarroum et al., 2015; Zhang Z.S. et al., 2016). Consistently, damage of PSI reaction center was actually alleviated by PSII photoinhibition in some extent, indicated by slower decrease of $\Delta \mathrm{MR} / \mathrm{MR}_{0}$ than $F_{\mathrm{v}} / F_{\mathrm{m}}$ and curtailed decrease in ratio of $\Delta \mathrm{MR} / \mathrm{MR}_{0}$ to $F_{\mathrm{v}} / F_{\mathrm{m}}$ since infection for 10 days (Figures $1 \mathrm{~B}-\mathrm{D}$ ). However, the positive role of PSII photoinhibition was limited for mitigating PSI damage, because of the great vulnerability of PSI to pathological wilting in apple seedlings infected by F. solani. 


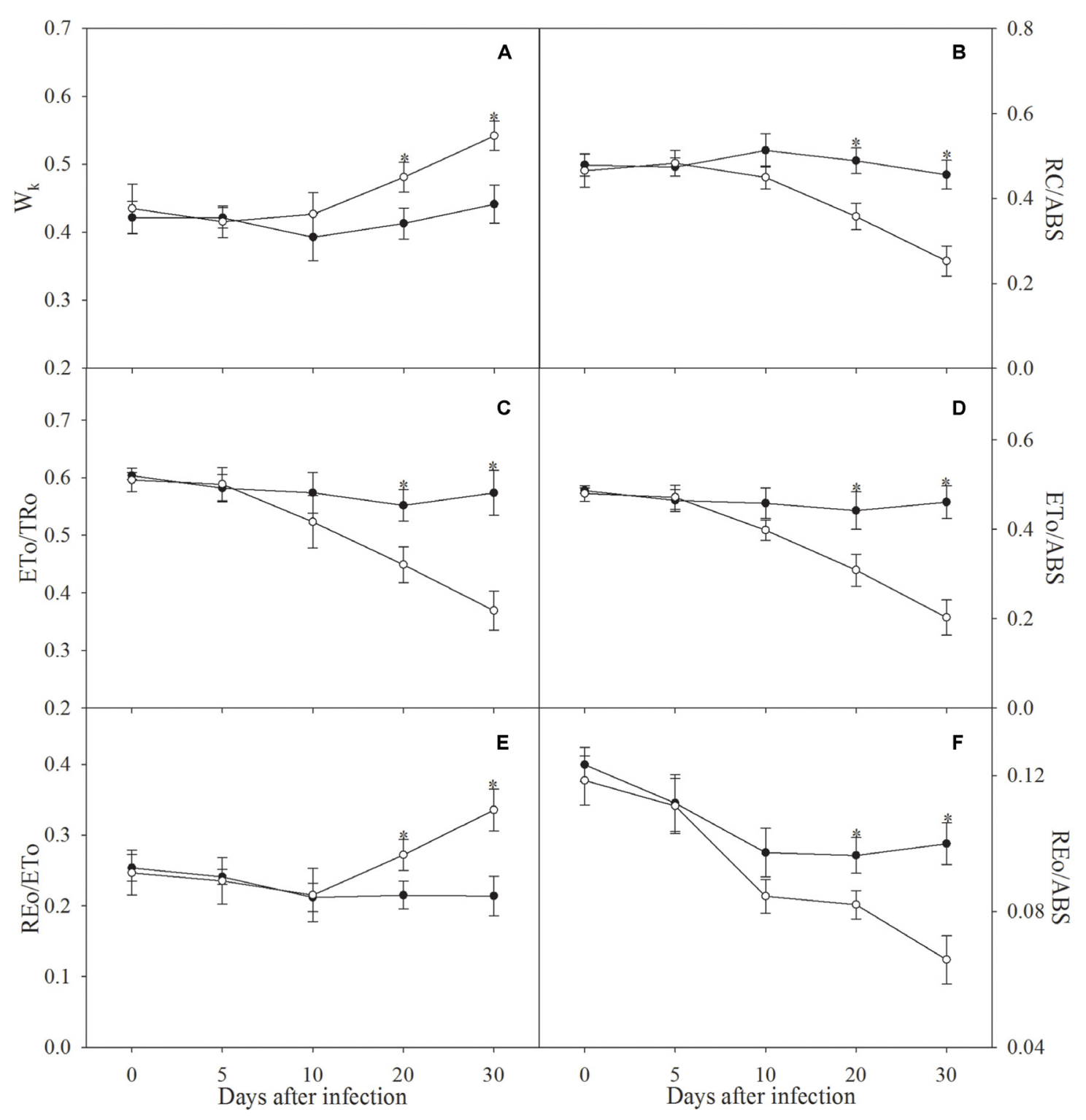

FIGURE $3 \mid W_{k}$ (A), RC/ABS (B), ETo/TRo (C), ETo/ABS (D), REo/ETo (E), and REo/ABS (F) in control plants (closed symbols) and plants infected by Fusarium solani (open symbols). The definition for these parameters is in Table 1. Data in the figure indicate the mean of five replicates ( \pm SD), and asterisk indicates significant difference at $P<0.05$.

In line with decreased amount of active PSII reaction centers (Figure 3B), photosynthetic electron transport beyond $\mathrm{Q}_{\mathrm{A}}$ was inhibited after 20 days of infection according to significant decrease of ETo/TRo and elevated J step (Figures 2A, 3C). Thus, the injury on PSII acceptors appeared (Schansker et al., 2005; Yan et al., 2013b). In contrast, greater injury occurred in OEC at PSII donor side, because a clear $\mathrm{K}$ step with significant increase of $W_{\mathrm{k}}$ appeared under inhibited electron transport beyond $\mathrm{Q}_{\mathrm{A}}$ (Figures 2A, 3A; Oukarroum et al., 2013, 2016b; Gao et al., 2014). These results indicated that leaf pathological wilting brought about deleterious effects on whole PSII components including reaction center, donor and acceptor sides. As a result, electron flow from PSII to PSI was greatly restricted (Figures 3D,F). Limited electron donation from PSII can promote PSI oxidation by retarding its re-reduction (Strasser et al., 2010; Goltsev et al., 2012; Yan et al., 2013a,b; Dabrowski et al., 2017), however, PSI oxidation after 10 days of infection was obviously inhibited (Figures 2B, 4A). Therefore, we inferred that inhibition on PSI photochemical capacity was greater compared with limited electron donation from PSII (Yan et al., 2015). This result conformed to above analysis about greater susceptibility of PSI reaction center, and unchanged ratio of PSI oxidation amplitude between infected plants and control plants might associated with the protective effect on PSI by declined PSII performance (Figure 4E). I step represents the kinetic bottleneck of electron transport chain due to limitation of 


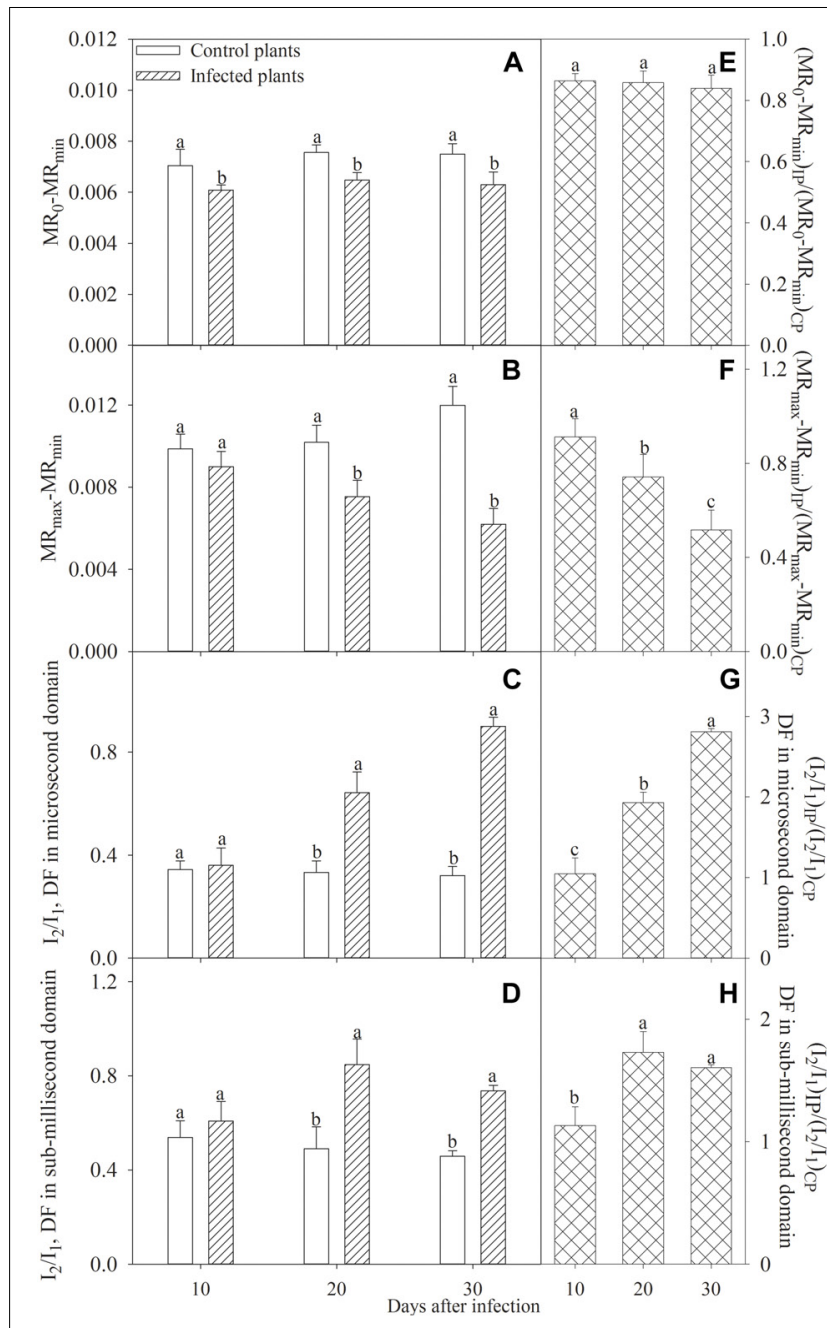

FIGURE 4 | MR $0-M_{\text {min }}$ (A), $M_{\text {max }}-M_{\text {min }}$ (B), $\mathrm{I}_{2} / \mathrm{I}_{1}$ in microsecond (C), and sub-millisecond (D) domains, and ratios of these parameters between plants infected by Fusarium solani and control plants (E-H). Data in the figure indicate the mean of five replicates $( \pm S D)$. Different letters on error bars indicate significant difference at $P<0.05$.

PQ re-oxidation (Schansker et al., 2005). Notably, slight change of I step and significant increase in REo/ETo after 20 days of infection indicated that PQ re-oxidation was not greatly affected, and the limitation on electron flow from PQ to PSI acceptors was lower than electron flux beyond $\mathrm{Q}_{\mathrm{A}}$ to PQ (Figures 2A, 3E). The elevated electron transport efficiency beyond PQ was also reported in Haberlea rhodopensis and Phaseolus vulgaris under water deficit stress (Strasser et al., 2010; Goltsev et al., 2012), however, this result seemed contradictory to damaged PSI reaction center. Notably, Zivcak et al. (2015) suggested that IP amplitude which also could reflect PQ re-oxidation efficiency depended on not only the activation of PSI reaction center but also electron transport capacity at PSI acceptor side. Thus, we supposed that the negative effect of damaged PSI reaction center on PQ re-oxidation could be compensated, because similar to declined PSII performance, inactivation of
PSI reaction center might protect PSI acceptors by reducing ROS production and consequently alleviated the reduction of electron transport efficiency beyond PQ. The positive role of inactivated PSI reaction center has been proposed recently and may be a specific response in apple to Fusarium-induced water deficit stress (Tiwari et al., 2016). After 20 days of infection, electron flow from PSII to PSI was blocked so seriously that PSI re-reduction could not be accomplished normally, leading to disconnection between PSII and PSI (Figures 2B, 4B). In parallel with increased $W_{\mathrm{k}}$ and decreased RC/ABS and ETo/TRo, the gradually decreased ratio of PSI re-reduction amplitude between infected plants and control plants also reflected aggravation of PSII damage during prolonged infection after 10 days (Figures 3A-C, 4F). In a word, susceptibility of PSI reaction center was confirmed in apple seedlings infected by F. solani through analyzing MR transients, and the disconnection between PSII and PSI was also apparently observed in these transients.

Delayed chlorophyll $a$ fluorescence in microsecond and submillisecond domains is mostly related to $\mathrm{Z}^{+} \mathrm{Q}_{\mathrm{A}}{ }^{-}$state of PS II (Goltsev et al., 2009). DF microsecond component is dominated by redox reactions at PSII donor side and can be affected by electron transport at PSII acceptor side, whereas DF sub-millisecond component which associates with activated PS II reaction center mainly depends on redox state of PSII acceptors (Goltsev et al., 2009). The occurrence of $I_{1}$ peak mainly results from accumulation of $\mathrm{S}_{3} \mathrm{Z}^{+} \mathrm{P}_{680 \mathrm{Q}_{\mathrm{A}}}{ }^{-}$state, as $\mathrm{S}_{3}$ state exhibits the highest DF signal during the $\mathrm{S}$-state cycle of OEC (Buchta et al., 2007; Schansker et al., 2011), and particularly, Oukarroum et al. (2016a) illustrated the relation between OEC injury and DF change. Thus, in accordance with elevated $\mathrm{K}$ step, remarkably lowered $\mathrm{I}_{1}$ in microsecond domain after 20 days infection also reflected the damage of OEC at PSII donor side (Figures 2A,C). $\mathrm{I}_{1}-\mathrm{I}_{2}-\mathrm{D}_{2}$ phase correlates with reduction process of $\mathrm{PQ}$ pool, and $\mathrm{I}_{2}$ is probably related to the prolonged reopening of PSII reaction centers by electron transfer from reduced $\mathrm{Q}_{\mathrm{B}}$ to $\mathrm{PQ}$ before full reduction of $\mathrm{PQ}$ pool (Goltsev et al., 2009; Strasser et al., 2010; Kalaji et al., 2012). Limited electron transport beyond $\mathrm{Q}_{\mathrm{A}}$ was beneficial to PSII reopening by inhibiting PQ reduction and could mitigate the decrease of microsecond DF, leading to significant increase of $\mathrm{I}_{2} / \mathrm{I}_{1}$ with less decrease in $\mathrm{I}_{2}$ than $\mathrm{I}_{1}$ after 20 days infection (Figures 2C, 4C). Thus, in agreement with the finding of Oukarroum et al. (2013), lowered $\mathrm{I}_{2}$ with increased $\mathrm{I}_{2} / \mathrm{I}_{1}$ in microsecond domain verified greater damage of PSII donor side than acceptor side, and the damage of PSII donor became more and more severe than acceptor side since infection for 10 days (Figure 4G). In contrast, significant increase of $I_{2}$ and $I_{2} / I_{1}$ in sub-millisecond domain depended on greater PQ re-oxidation than PQ reduction after 20 days infection (Figures 2D, 4D), and in other words, electron transport beyond PQ was less affected than electron donation from PSII to PQ, which was consistent with the elevated REo/ETo (Figure 3E). In accordance with MR transients, the disconnection of PSII and PSI due to remarkably lowered electron donation from PSII was also reflected by the increased ratio of $\mathrm{I}_{2} / \mathrm{I}_{1}$ in sub-millisecond domain between infected seedlings and control seedlings as well as the 
elevated $\mathrm{DF}$ transients from $\mathrm{I}_{2}$ to $\mathrm{D}_{2}$ after 20 days infection (Figures 2B,D, 4H). Obviously, the data from DF, PF and MR can be validated reciprocally.

In agreement with the hypothesis, infection of $F$. solani depressed PSII and PSI performance and destroyed their coordination in apple seedlings by inducing pathological foliage wilting. PSI was more susceptible to infection of $F$. solani than PSII, although depression of PSII performance aided in ameliorating PSI injury to some extent.

\section{AUTHOR CONTRIBUTIONS}

KY designed the experiment, performed the data analysis, and wrote the manuscript. GH and CR participated in the experiment design and polished the language. SZ reviewed the manuscript

\section{REFERENCES}

Ashraf, M., and Foolad, M. R. (2007). Roles of glycine betaine and proline in improving plant abiotic stress resistance. Environ. Exp. Bot. 59, 206-216. doi: 10.1016/j.envexpbot.2005.12.006

Buchta, J., Grabolle, M., and Dau, H. (2007). Photosynthetic dioxygen formation studied by time-resolved delayed fluorescence measurements - Method, rationale, and results on the activation energy of dioxygen formation. Biochim. Biophys. Acta 1767, 565-574. doi: 10.1016/j.bbabio.2007. 04.003

Campos, H., Trejo, C., Pena-Valdivia, C. B., Garcia-Nava, R., Conde-Martinez, F., and Cruz-Ortega, M. D. (2014). Photosynthetic acclimation to drought stress in Agave salmiana Otto ex Salm-Dyck seedlings is largely dependent on thermal dissipation and enhanced electron flux to photosystem I. Photosynth. Res. 122, 23-39. doi: 10.1007/s11120-014-0008-6

Dabrowski, P., Kalaji, M. H., Baczewska, A. H., Pawluskiewicz, B., Mastalerczuk, G., Borawska-Jarmulowicz, B., et al. (2017). Delayed chlorophyll a fluorescence, MR820, and gas exchange changes in perennial ryegrass under salt stress. J. Lumin. 183, 322-333. doi: 10.1016/j.jlumin.2016.11.031

Dong, X., Wang, M., Ling, N., Shen, Q. R., and Guo, S. W. (2016). Potential role of photosynthesis-related factors in banana metabolism and defense against Fusarium oxysporum f. sp cubense. Environ. Exp. Bot. 129, 4-12. doi: 10.1016/j. envexpbot.2016.01.005

Gao, J., Li, P., Ma, F., and Goltsev, V. (2014). Photosynthetic performance during leaf expansion in Malus micromalus probed by chlorophyll a fluorescence and modulated 820nm reflection. J. Photochem. Photobiol. B Biol. 137, 144-150. doi: 10.1016/j.jphotobiol.2013.12.005

Goltsev, V., Zaharieva, I., Chernev, P., Kouzmanova, M., Kalaji, H. M., Yordanov, I., et al. (2012). Drought-induced modifications of photosynthetic electron transport in intact leaves: analysis and use of neural networks as a tool for a rapid non-invasive estimation. Biochim. Biophys. Acta 1817, 1490-1498. doi: 10.1016/j.bbabio.2012.04.018

Goltsev, V., Zaharieva, I., Chernev, P., and Strasser, R. J. (2009). Delayed fluorescence in photosynthesis. Photosynth. Res. 101, 217-232. doi: 10.1007/ s11120-009-9451-1

Henfrey, J. L., Baab, G., and Schmitz, M. (2015). Physiological stress responses in apple under replant conditions. Sci. Hortic. 194, 111-117. doi: 10.1016/j.scienta. 2015.07.034

Huang, W., Fu, P. L., Jiang, Y. J., Zhang, J. L., Zhang, S. B., Hu, H., et al. (2013). Differences in the responses of photosystem I and photosystem II of three tree species Cleistanthus sumatranus, Celtis philippensis and Pistacia weinmannifolia exposed to a prolonged drought in a tropical limestone forest. Tree Physiol. 33, 211-220. doi: 10.1093/treephys/tps132

Kalaji, H. M., Goltsev, V., Bosa, K., Allakhverdiev, S. I., Strasser, R. J., and Govindjee (2012). Experimental in vivo measurements of light emission in plants: a perspective dedicated to David Walker. Photosynth. Res. 114, 69-96. doi: $10.1007 / \mathrm{s} 11120-012-9780-3$ and proposed some critical suggestions. XW and TB participated in the experiment and data analysis.

\section{FUNDING}

This research was jointly supported by Shandong Provincial Natural Science Foundation, China (ZR2017QC005), Yantai Science and Technology Planning Project (2015ZH069; 2016ZH074), Key Deployment Project of Chinese Academy of Sciences (KFZD-SW-112), the Science and Technology Service Network Initiative (KFJ-STS-ZDTP-023), Opening Foundation of the State Key Laboratory of Crop Biology, Shandong Agricultural University (2016KF07), and Young Scientists Partner Project between Shenyang Branch of Chinese Academy of Sciences and Shandong Academy of Sciences.

Mazzola, M., and Manici, L. M. (2012). “Apple replant disease: role of microbial ecology in cause and control," in Annual Review of Phytopathology, eds N. K. VanAlfen, J. E. Leach, and S. Lindow (Palo Alto, CA: Annual Reviews), 45-65.

Nogues, S., Cotxarrera, L., Alegre, L., and Trillas, M. I. (2002). Limitations to photosynthesis in tomato leaves induced by Fusarium wilt. New Phytol. 154, 461-470. doi: 10.1046/j.1469-8137.2002.00379.x

Oukarroum, A., Bussotti, F., Goltsev, V., and Kalaji, H. M. (2015). Correlation between reactive oxygen species production and photochemistry of photosystems I and II in Lemna gibba L. plantsunder salt stress. Environ. Exp. Bot. 109, 80-88. doi: 10.1016/j.envexpbot.2014.08.005

Oukarroum, A., Goltsev, V., and Strasser, R. J. (2013). Temperature effects on pea plants probed by simultaneous measurements of the kinetics of prompt fluorescence, delayed fluorescence and modulated $820 \mathrm{~nm}$ reflection. PLoS One 8:10. doi: 10.1371/journal.pone.0059433

Oukarroum, A., Gharous, M. E., Goltsev, V., and Strasser, R. J. (2016a). Delayed fluorescence emission as a probe for the response of photosynthetic organisms to high temperature exposure: a comparative study. J. Lumin. 180, 321-327. doi: 10.1016/j.jlumin.2016.08.061

Oukarroum, A., Madidi, S. E., and Strasser, R. J. (2016b). Differential heat sensitivity index in barley cultivars (Hordeum vulgare L.) monitored by chlorophyll a fluorescence OKJIP. Plant Physiol. Biochem. 105, 102-108. doi: 10.1016/j.plaphy.2016.04.015

Pshibytko, N. L., Zenevich, L. A., and Kabashnikova, L. F. (2006). Changes in the photosynthetic apparatus during fusarium wilt of tomato. Russ. J. Plant Physiol. 53, 25-31. doi: 10.1134/S1021443406010031

Schansker, G., Srivastava, A., Govindjee, and Strasser, R. J. (2003). Characterization of the 820-nm transmission signal paralleling the chlorophyll a fluorescence rise (OJIP) in pea leaves. Funct. Plant Biol. 30, 785-796. doi: 10.1071/FP03032

Schansker, G., Toth, S. Z., Kovacs, L., Holzwarth, A. R., and Garab, G. (2011). Evidence for a fluorescence yield change driven by a light-induced conformational change within photosystem II during the fast chlorophyll a fluorescence rise. Biochim. Biophys. Acta 1807, 1032-1043. doi: 10.1016/j. bbabio.2011.05.022

Schansker, G., Toth, S. Z., and Strasser, R. J. (2005). Methylviologen and dibromothymoquinone treatments of pea leaves reveal the role of photosystem I in the Chl a fluorescence rise OJIP. Biochim. Biophys. Acta 1706, 250-261. doi: 10.1016/j.bbabio.2004.11.006

Sonoike, K. (2011). Photoinhibition of photosystem I. Physiol. Plant 142, 56-64. doi: 10.1111/j.1399-3054.2010.01437.x

Strasser, R. J., Tsimilli-Michael, M., Qiang, S., and Goltsev, V. (2010). Simultaneous in vivo recording of prompt and delayed fluorescence and $820 \mathrm{~nm}$ reflection changes during drying and after rehydration of the resurrection plant Haberlea rhodopensis. Biochim. Biophys. Acta 1797, 122-122. doi: 10.1016/j.bbabio.2010. 03.008

Takahashi, S., and Murata, N. (2008). How do environmental stresses accelerate photoinhibition? Trends Plant Sci. 13, 178-182. doi: 10.1016/j.tplants.2008. 01.005 
Tewoldemedhin, Y. T., Mazzola, M., Botha, W. J., Spies, C. F. J., and McLeod, A. (2011a). Characterization of fungi (Fusarium and Rhizoctonia) and oomycetes (Phytophthora and Pythium) associated with apple orchards in South Africa. Eur. J. Plant Pathol. 130, 215-229. doi: 10.1007/s10658-0119747-9

Tewoldemedhin, Y. T., Mazzola, M., Labuschagne, I., and McLeod, A. (2011b). A multi-phasic approach reveals that apple replant disease is caused by multiple biological agents, with some agents acting synergistically. Soil Biol. Biochem. 43, 1917-1927. doi: 10.1016/j.soilbio.2011.05.014

Tewoldemedhin, Y. T., Mazzola, M., Mostert, L., and McLeod, A. (2011c). Cylindrocarpon species associated with apple tree roots in South Africa and their quantification using real-time PCR. Eur. J. Plant Pathol. 129, 637-651. doi: 10.1007/s10658-010-9728-4

Tiwari, A., Mamedov, F., Grieco, M., Suorsa, M., Jajoo, A., Styring, S., et al. (2016). Photodamage of iron-sulphur clusters in photosystem I induces nonphotochemical energy dissipation. Nat. Plants 2:16035. doi: $10.1038 /$ nplants. 2016.35

Wang, M., Sun, Y., Sun, G., Liu, X., Zhai, L., Shen, Q., et al. (2015). Water balance altered in cucumber plants infected with Fusarium oxysporum f. sp. cucumerinum. Sci. Rep. 5:7722. doi: 10.1038/srep07722

Wang, Y. F., Pan, F. B., Wang, G. S., Zhang, G. D., Wang, Y. L., Chen, X. S., et al. (2014). Effects of biochar on photosynthesis and antioxidative system of Malus hupehensis Rehd. seedlings under replant conditions. Sci. Hortic. 175, 9-15. doi: 10.1016/j.scienta.2014.05.029

Yan, K., Chen, P., Shao, H., Zhao, S., Zhang, L., Zhang, L., et al. (2012b). Responses of photosynthesis and photosystem II to higher temperature and salt stress in sorghum. J. Agron. Crop Sci. 198, 218-226. doi: 10.1111/j.1439-037X.2011. 00498.x

Yan, K., Chen, P., Shao, H., Zhao, S., Zhang, L., Zhang, L., et al. (2012a). Photosynthetic characterization of Jerusalem artichoke during leaf expansion. Acta Physiol. Plant. 34, 353-360. doi: 10.1007/s11738-011-0834-5

Yan, K., Chen, P., Shao, H. B., Shao, C. Y., Zhao, S. J., and Brestic, M. (2013a). Dissection of photosynthetic electron transport process in sweet sorghum under heat stress. PLoS One 8:e62100. doi: 10.1371/journal.pone.0062100

Yan, K., Chen, P., Shao, H. B., and Zhao, S. J. (2013b). Characterization of photosynthetic electron transport chain in bioenergy crop Jerusalem artichoke (Helianthus tuberosus L.) under heat stress for sustainable cultivation. Ind. Crop Prod. 50, 809-815. doi: 10.1016/j.indcrop.2013.08.012

Yan, K., Wu, C., Zhang, L., and Chen, X. (2015). Contrasting photosynthesis and photoinhibition in tetraploid and its autodiploid honeysuckle (Lonicera japonica Thunb.) under salt stress. Front. Plant Sci. 6:227. doi: 10.3389/fpls. 2015.00227

Yang, S. M., Li, X., Chen, W. Q., Liu, T. G., Zhong, S. F., Ma, L. X., et al. (2016). Wheat resistance to fusarium head blight is associated with changes in photosynthetic parameters. Plant Dis. 100, 847-852. doi: 10.1094/PDIS-04-140398-RE
Yim, B., Smalla, K., and Winkelmann, T. (2013). Evaluation of apple replant problems based on different soil disinfection treatments-links to soil microbial community structure? Plant Soil 366, 617-631. doi: 10.1007/s11104-0121454-6

Yin, C., Xiang, L., Wang, G., Wang, Y., Shen, X., Chen, X., et al. (2017). Phloridzin promotes the growth of Fusarium moniliforme (Fusarium verticillioides). Sci. Hortic. 214, 187-194. doi: 10.1016/j.scienta.2016.11.035

Zhang, S. B., Huang, W., Zhang, J. L., and Cao, K. F. (2016). Differential responses of photosystems I and II to seasonal drought in two Ficus species. Acta Oecol. 73, 53-60. doi: 10.1016/j.actao.2016.03.001

Zhang, Z. S., Jin, L. Q., Li, Y. T., Tikkanen, M., Li, Q. M., Ai, X. Z., et al. (2016). Ultraviolet-B radiation (UV-B) relieves chilling-light-induced PSI photoinhibition and accelerates the recovery of CO2 assimilation in cucumber (Cucumis sativus L.) leaves. Sci. Rep. 6:34455. doi: 10.1038/srep34455

Zivcak, M., Brestic, M., Kalaji, H. M., and Govindjee (2014a). Photosynthetic responses of sun- and shade-grown barley leaves to high light: is the lower PSII connectivity in shade leaves associated with protection against excess of light? Photosynth. Res. 119, 339-354. doi: 10.1007/s11120-014-9969-8

Zivcak, M., Brestic, M., Kunderlikova, K., Olsovska, K., and Allakhverdiev, S. I. (2015). Effect of photosystem I inactivation on chlorophyll a fluorescence induction in wheat leaves: does activity of photosystem I play any role in OJIP rise? J. Photochem. Photobiol. B Biol. 152, 318-324. doi: 10.1016/j.jphotobiol. 2015.08.024

Zivcak, M., Brestic, M., Olsovska, K., and Slamka, P. (2008). Performance index as a sensitive indicator of water stress in Triticum aestivum L. Plant Soil Environ. 54, 133-139. doi: 10.17221/392-PSE

Zivcak, M., Kalaji, H. M., Shao, H. B., Olsovska, K., and Brestic, M. (2014b). Photosynthetic proton and electron transport in wheat leaves under prolonged moderate drought stress. J. Photochem. Photobiol. B Biol. 137, 107-115. doi: 10.1016/j.jphotobiol.2014.01.007

Zivcak, M., Olsovska, K., Slamka, P., Galambosova, J., Rataj, V., Shao, H. S., et al. (2014c). Application of chlorophyll fluorescence performance indices to assess the wheat photosynthetic functions influenced by nitrogen deficiency. Plant Soil Environ. 60, 210-215. doi: 10.17221/73/2014-PSE

Conflict of Interest Statement: The authors declare that the research was conducted in the absence of any commercial or financial relationships that could be construed as a potential conflict of interest.

Copyright (c) 2018 Yan, Han, Ren, Zhao, Wu and Bian. This is an open-access article distributed under the terms of the Creative Commons Attribution License (CC BY). The use, distribution or reproduction in other forums is permitted, provided the original author(s) and the copyright owner are credited and that the original publication in this journal is cited, in accordance with accepted academic practice. No use, distribution or reproduction is permitted which does not comply with these terms. 